\title{
A Mixed TS-ISA Algorithm for Reliability Redundancy Optimization Problem
}

\author{
Zhu Haiying1, Liu Yubao ${ }^{2}$ \\ ${ }^{1}$ Information Technology Branch Changchun Vocational Institute of Technology, \\ Changchun, China \\ ${ }^{2}$ College of Computer Science and Technology, \\ Changchun University, Changchun, China \\ 137132478@qq.com
}

\begin{abstract}
This article is based on the mixture of tabu search algorithm and interior search algorithm(ISA) to address the reliability redundancy assignment problem. Iinterior search algorithm is immersed in a tabu search algorithm. TS is used to search solution space and ISA is applied to generate neighborhood solutions. The merit of two algorithms is considered at the same time. And a mixed TS-ISA method is proposed to deal with three benchmark reliability redundancy optimization problem. The experimental results show that a the method is effective and efficient for RRAP by comparing with other results in the previous literaturs.
\end{abstract}

Keywords: Tabu search, interior search algorithm, reliability redundancy allocation, nonlinear programming

\section{Introduction}

Reliability optimization problem is very significant in the field of industrial engineering and academic. In general, there are two main ways to improve the reliability of the system. The first way is to increase the components' reliability, and the second approach is to useredundant components of the subsystem. In the first approach, sometimes it can not satisfy our demand although you use the current best reliable components. The other way is to select the components combination to reach the highest level system reliability. However the volume, cost and weight are increased. It is essential to balance these two options. Such as reliability and redundancy assignment problem is called RRAP[1].

Reliability redundancy assignment problem of nonlinear constraints [4] belongs to the mixed integer programming subject. It can be described uniformly as follows:

$\operatorname{Max} R_{s}=f(r, n)$

s.t. $\mathrm{g}_{\mathrm{j}}(\mathrm{r}, \mathrm{n}) \leq \mathrm{b}_{\mathrm{j}}, \mathrm{j}=1 . . \mathrm{m} ; \mathrm{n}_{\mathrm{j}} \in$ positive integer, $0 \leq \mathrm{r}_{\mathrm{j}} \leq 1$

RRAP is proven to be NP-hard problem. Many different optimization approaches were proposed to solve it. These methods are called meta-heuristics methods. They are widely researched and used. They can get feasible solutions within limited computing time. Many researcher [2-7] use some algorithms to solve the problem of the reliability design of the series-parallel, complex (bridge) and overspeed protection system. Lately some mixed meta-heuristic method are put forward to solve the RRAP[8-10].

There are generally some defects though the above methods can gain near optimal solutions in reasonable time, such as convergence velocity and accuracy are slow et al. In addition, the balance of accuracy and convergence velocity are not thinked.

This paper proposed a mixed tabu search and interior search algorithm(ISA). ISA has 
fast convergence speed. On the other hand, memory function of TS is very strong, it can achieve higher precision of solution. But its convergence velocity is slow. The proposed method considered the merit of both methods. It is applied to resolve three problems in the RRAP. Experimental results demonstrate that the method has better performance on accuracy and convergence velocity for RRAP.

\section{The Mixed Algorithm based on TS and ISA}

\subsection{Tabu Search Approach}

Tabu search(TS) is a meta-heuristic method. It has become the preferred method to solve many complex optimization problems. It couples the local searching with the tabu list to prevent finding the same solution. It is very effective for solving many optimization problems.

Tabu search is an iterative process. In each iteration, it moves from the current address to new neighborhood solutions. The method is described as follows:

Step 1. Generated randomly the initial solution. Initializing parameters. The null value is given to Tabu list.

Step 2. Checking the stop condition. If satisfied, search is stopped and is to accepting the current best solution. Otherwise continuing the next steps.

Step 3. Producing neighborhood solution, and selecting a number of candidate solutions.

Step 4. Checking the aspiration condition. Standard inspection. If a candidate solution meets the aspiration condition, it will be accepted the new current optimal solution, and return to step 2. Or else continue the next steps.

Step 5. The best state is selected from the candidate solution. It will be current the optimal solution, and the tabu list is updated.

Step 6. Return step 2.

\subsection{Interior Search Algorithm}

The interior search algorithm(ISA) [16] is a proposing metaheuristic algorithm proposed by Amir H. Gandomi in 2014.It is different from another optimization algorithm called the interior point method in the literature.The algorithm is inspired by construction process mentioned in the previous section. In this algorithm, in addition to the fittest element, the other elements are divided into two groups. One group called the composition groups, the composition of elements are changed to find a more beautiful view. Another one called the mirror groups, mirrors are placed between these elements and the fittest elements in order to get better views. The algorithm is described as follows:

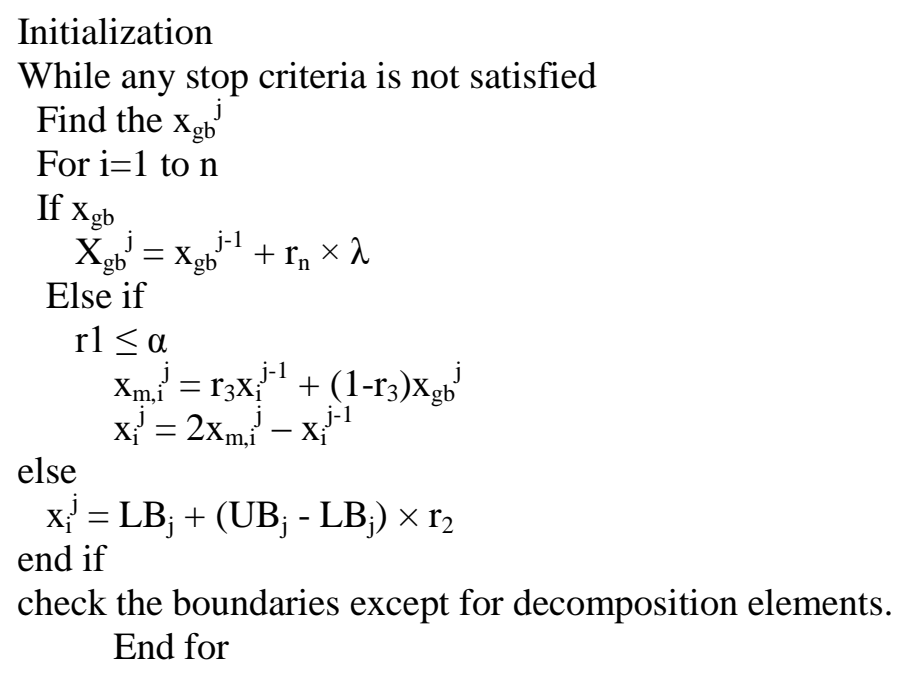




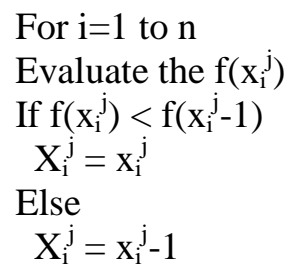

End for

End while

\section{The Mixed TS-ISA Algorithm}

ISA algorithm is a good method for many optimization problem. But it has disadvantages such as low accuracy, and easy to get divergence solution. TS can increase to find an optimal solution. But TS maybe waste resources for poor individual with no select operator. In order to improve the convergence velocity and get better quality solutions, ISA algorithm is absorbed by tabu search algorithm. TS is used to search solution space and ISA is used to generate neighborhood solutions. Thus TS can help to get a better solution in each generation of the ISA algorithm, and get the current best solution throughout aspiration condition. The proposed algorithm consider the merit of both methods at the same time.

The mixed algorithm is described as follows:

Step 1. Parameters Initialized. The null value is given to Tabu list.

Step 2. Initializing randomly original population $\mathrm{x}$, generating the current most optimal solution $\mathrm{x}_{\text {best }}$.

Step 3. Checkng the stop condition. If it is met, searching is stopped and the current most optimal solution is got. Or else continue the next steps.

Step 4. The neighborhood solutions are generated by using ISA algorithm. And selecting the best solution from the current candidate solutions.

Step 5. Checking the aspiration condition. If a candidate solution meets the aspiration condition, it is selected as the new current most optimal solution, and then return step 2. Or else continue the next steps.

Step 6. Selecting the best one from the candidate solution as the current most optimal solution, at the same time updating the tabu list. And Go step3.

The main processing of generating neighborhood solution by the ISA algorithm is described as follows:

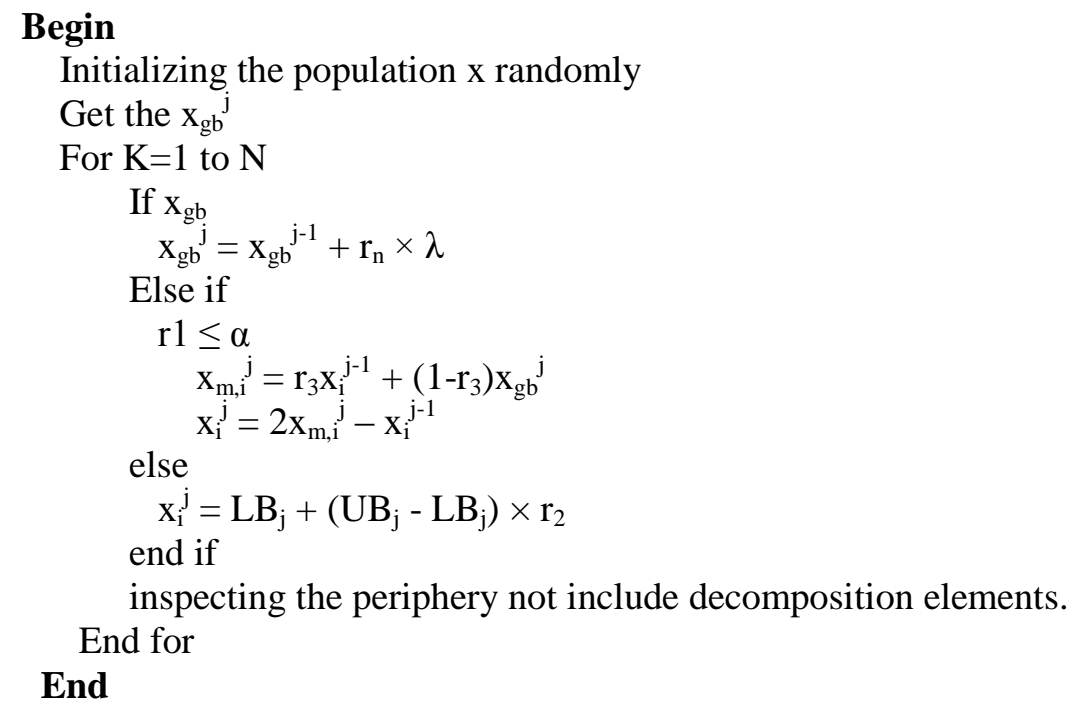

Choosing the best solution from this generation candidate solutions 


\section{Simulations and Comparisons}

In this section, we developed simulation on three benchmark RRAP for testing performance of TS-ISA. We compared the result of TS-ISA with that of some other algorithms reported in literatures.

The penalty function is applied for dealing with constraints, it is:

$$
\min F(x)=-f(x)+\lambda \sum_{j=1}^{p} \max \left\{O, g_{j}(x)\right\}^{2}
$$

Where $F(x)$ is penalty function and $f(x)$ is objective function. $g_{j}(x),(j=1,2, p)$ is the $j$ th constraint. $\lambda$ is called penalty coefficient.

\subsection{Problem1: Series-Parallel System}

Series-parallel system [11][12][4] is shown in Figure 1:

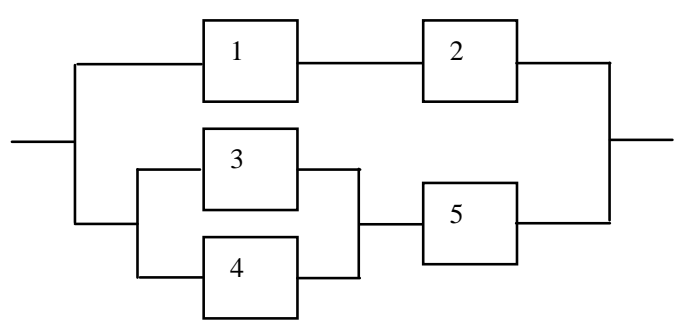

Figure 1. Series-Parallel System

This problem is described as follows:

$\operatorname{Max} f(r, n)=1-\left(1-R_{1} R_{2}\right)\left(1-\left(1-\left(1-R_{3}\right)\left(1-R_{4}\right)\right) R_{5}\right)$

$$
\begin{array}{ll}
\text { s.t. } & \mathrm{g}_{1}(\mathrm{r}, \mathrm{n})=\sum_{\mathrm{i}=1}^{\mathrm{m}} \mathrm{w}_{\mathrm{i}} \mathrm{v}_{\mathrm{i}}{ }^{2} \mathrm{n}_{\mathrm{i}}{ }^{2} \leq \mathrm{V} \\
\mathrm{g}_{2}(\mathrm{r}, \mathrm{n})=\sum_{\mathrm{i}=1}^{\mathrm{m}} \alpha_{\mathrm{i}}\left(-1000 / \ln \mathrm{r}_{\mathrm{i}}\right)^{\beta_{\mathrm{i}}}\left(\mathrm{n}_{\mathrm{i}}+\exp \left(\mathrm{n}_{\mathrm{i}} / 4\right)\right) \leq \mathrm{C} \\
\left.\mathrm{g}_{3}(\mathrm{r}, \mathrm{n})=\sum_{\mathrm{i}=1}^{\mathrm{m}} \mathrm{w}_{\mathrm{i}} \mathrm{n}_{\mathrm{i}} \exp \left(\mathrm{n}_{\mathrm{i}} / 4\right)\right) \leq \mathrm{W}
\end{array}
$$

The problem of parameters are shown in Table 1:

Table 1. The Parameters of Series-Parallel System

\begin{tabular}{|c|c|c|c|c|c|c|c|}
\hline Subsystem $\mathrm{i}$ & $10^{5} \mathrm{\alpha}_{\mathrm{i}}$ & $\beta_{\mathrm{i}}$ & $\mathrm{W}_{\mathrm{i} \mathrm{v}_{\mathrm{i}}{ }^{2}}$ & $\mathrm{w}_{\mathrm{i}}$ & $\mathrm{V}$ & $\mathrm{C}$ & $\mathrm{W}$ \\
\hline 1 & 2.500 & 1.5 & 2 & 3.5 & 180 & 175 & 100 \\
\hline 2 & 1.450 & 1.5 & 4 & 4.0 & & & \\
\hline 3 & 0.541 & 1.5 & 5 & 4.0 & & & \\
\hline 4 & 0.541 & 1.5 & 8 & 3.5 & & & \\
\hline 5 & 2.100 & 1.5 & 4 & 4.5 & & & \\
\hline
\end{tabular}

\subsection{P2: Complex (Bridge) System}

Complex (bridge) system [13] [14] is described as Figure.2:

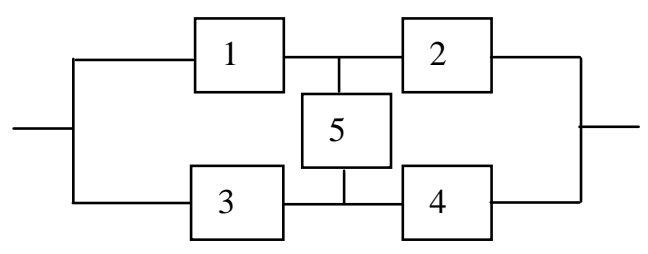

Figure 2. Complex (Bridge) System 
The formula is:

$$
\begin{array}{cc}
\operatorname{Max} & f(r, n)=R_{1} R_{2}+R_{3} R_{4}+R_{1} R_{4} R_{5}+R_{2} R_{3} R_{5} \\
& -R_{1} R_{2} R_{3} R_{4}-R_{1} R_{2} R_{3} R_{5}-R_{1} R_{2} R_{4} R_{5} \\
& -R_{1} R_{3} R_{4} R_{5}-R_{2} R_{3} R_{4} R_{5}+2 R_{1} R_{2} R_{3} R_{4} R_{5}
\end{array}
$$

The parameters are shown in Table 2:

Table 2. The Parameters of Complex (Bridge) System

\begin{tabular}{|l|l|l|l|l|l|l|l|}
\hline Subsystem $\mathrm{i}$ & $10^{5} \alpha_{\mathrm{i}}$ & $\beta_{\mathrm{i}}$ & $\mathrm{w}_{\mathrm{i}} \mathrm{v}_{\mathrm{i}}^{2}$ & $\mathrm{~W}_{\mathrm{i}}$ & $\mathrm{V}$ & $\mathrm{C}$ & $\mathrm{W}$ \\
\hline 1 & 2.33 & 1.5 & 1 & 7 & 110 & 175 & 200 \\
\hline 2 & 1.450 & 1.5 & 2 & 8 & & & \\
\hline 3 & 0.541 & 1.5 & 3 & 8 & & & \\
\hline 4 & 8.050 & 1.5 & 4 & 6 & & & \\
\hline 5 & 1.950 & 1.5 & 2 & 9 & & & \\
\hline
\end{tabular}

\subsection{Problem 3: Overspeed Protection System}

This problem is applied for protecting overspeed of gas turbine. The system will stop [15] when overspeed occurs. This system is shown in Figure 3:

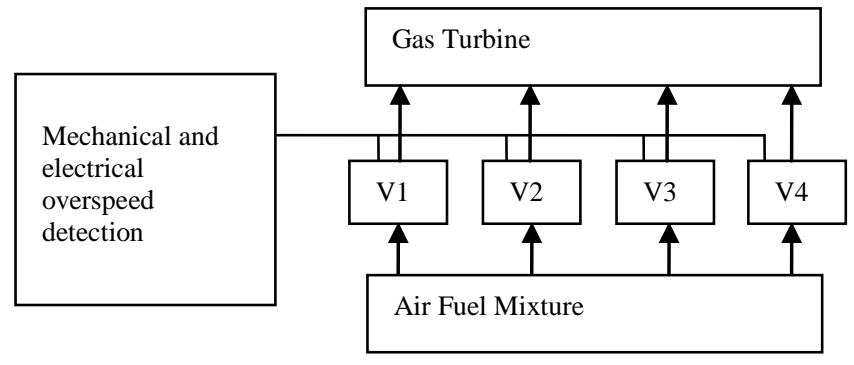

Figure 3. The Overspeed Protection System of a Gas Turbine

It is formulated (5):

$$
\begin{aligned}
& \text { Max } \mathrm{f}(\mathrm{r}, \mathrm{n})=\prod_{\mathrm{i}=1}^{\mathrm{m}}\left[1-\left(1-\mathrm{r}_{\mathrm{i}}\right)^{\mathrm{n}_{\mathrm{i}}}\right] \\
& \text { s.t. } \mathrm{h}_{1}(\mathrm{r}, \mathrm{n})=\sum_{\mathrm{i}=1}^{\mathrm{m}} \mathrm{v}_{\mathrm{i}} \mathrm{n}_{\mathrm{i}}{ }^{2} \leq \mathrm{V} \\
& \mathrm{h}_{2}(\mathrm{r}, \mathrm{n})=\sum_{\mathrm{i}=1}^{\mathrm{m}} \mathrm{C}\left(\mathrm{r}_{\mathrm{i}}\right) \cdot\left[\mathrm{n}_{\mathrm{i}}+\exp \left(\mathrm{n}_{\mathrm{i}} / 4\right)\right] \leq \mathrm{C} \\
& \mathrm{h}_{3}(\mathrm{r}, \mathrm{n})=\sum_{\mathrm{i}=1}^{\mathrm{m}} \mathrm{w}_{\mathrm{i}} \mathrm{n}_{\mathrm{i}} \exp \left(\mathrm{n}_{\mathrm{i}} / 4\right) \leq \mathrm{W} \\
& 1.0 \leq \mathrm{n}_{\mathrm{i}} \leq 10, \mathrm{n}_{\mathrm{i}} \in \mathrm{Z}^{+} \\
& 0.5 \leq \mathrm{r}_{\mathrm{i}} \leq 1-10^{-6}, \mathrm{r}_{\mathrm{i}} \in \mathrm{R}^{+}
\end{aligned}
$$

Here $C\left(r_{i}\right)=\alpha_{i}\left(-T / \ln r_{i}\right)^{\beta_{i}}, \mathrm{~T}$ is the task time.

The values of the parameters are shown in Table 3:

Table 3. The Parameters of Overspeed Protection System

\begin{tabular}{|c|c|c|c|c|c|c|c|c|}
\hline Subsystem i & $\begin{array}{c}10 \\
5 \alpha \mathrm{i}\end{array}$ & $\beta \mathrm{i}$ & $\mathrm{vi}$ & $\begin{array}{c}\mathrm{w} \\
\mathrm{i}\end{array}$ & $\mathrm{V}$ & $\mathrm{C}$ & $\mathrm{W}$ & $\mathrm{T}$ \\
\hline 1 & 1 & 1.5 & 1 & 6 & 250 & 400 & 500 & 1000 \\
\hline 2 & 2.3 & 1.5 & 2 & 6 & & & & \\
\hline 3 & 0.3 & 1.5 & 3 & 8 & & & & \\
\hline 4 & 2.3 & 1.5 & 2 & 7 & & & & \\
\hline
\end{tabular}

For analyzing performance of ISA, TS and TS-ISA. The simulation are developed for 
above three benchmark problems. For TS, the maximum count of iterations is 5000 , the size of the tabu list is 24 . For ISA algorithm, $\alpha=0.3$, the size of population $M=50$, the largest number of iterations is set to 5000. Each method running 50 times for each problem independly. The results are shown in table 4, table 5 and table 6 . best denotes the best value, worst denotes the worst value, mean denotes average value and SD denotes standard deviation.

Table 4. The Results of the Series Parallel System using Three Algorithms

\begin{tabular}{|c|c|c|c|c|}
\hline Algorithm & Best & Worst & Mean & SD \\
\hline TS & 0.999972 & 0.992831 & 0.999160 & $1.3553 \mathrm{e}-03$ \\
& 53 & 46 & 67 & \\
\hline ISA & 0.999898 & 0.983580 & 0.997535 & $2.8176 \mathrm{e}-03$ \\
& 44 & 39 & 64 & \\
\hline TS- ISA & 0.999976 & 0.999964 & 0.999976 & $1.8994 \mathrm{e}-06$ \\
& 65 & 76 & 28 & \\
\hline
\end{tabular}

Table 5. The Results of the Complex (Bridge) System using Three Algorithms

\begin{tabular}{|c|c|c|c|c|}
\hline $\begin{array}{c}\text { Algorith } \\
\mathrm{m}\end{array}$ & Best & Worst & Mean & SD \\
\hline TS & 0.99982818 & 0.96688487 & 0.99496166 & $\begin{array}{c}7.3952 \mathrm{e} \\
-03\end{array}$ \\
\hline ISA & 0.99957392 & 0.96176638 & 0.99123325 & $\begin{array}{c}9.6013 \mathrm{e} \\
-03\end{array}$ \\
\hline TS- ISA & 0.99988964 & 0.99988935 & 0.99988944 & $\begin{array}{c}1.3290 \mathrm{e} \\
-07\end{array}$ \\
\hline
\end{tabular}

Table 6. The Results of the Overspeed Protection System using Three Algorithms

\begin{tabular}{|c|c|c|c|c|}
\hline Algorithm & Best & Worst & Mean & SD \\
\hline TS & 0.99991922 & 0.91363947 & 0.984195 & $2.2715 \mathrm{e}$ \\
& & & 01 & -02 \\
\hline ISA & 0.99881031 & 0.95239924 & 0.987674 & $1.1077 \mathrm{e}$ \\
& & & 42 & -02 \\
\hline TS- ISA & 0.99995467 & 0.99994615 & 0.999954 & $1.2504 \mathrm{e}$ \\
& & & 50 & -06 \\
\hline
\end{tabular}

Figure 4, Figure 5 and Figure 6 Figure 5 show optimization curve which use three algorithms to solve three benchmark RRAP:

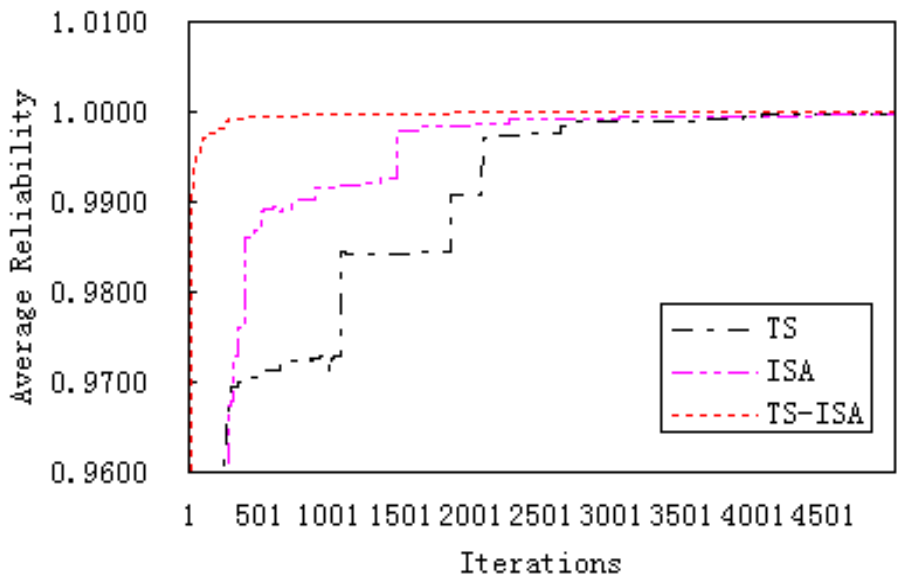

Figure 4. Series-Parallel System 


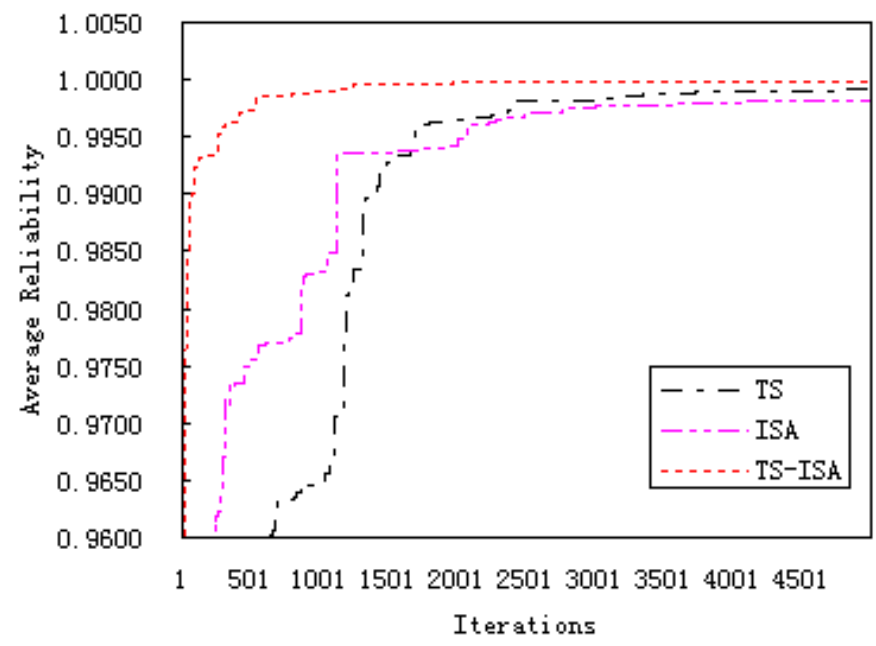

Figure 5. Complex (Bridge) System

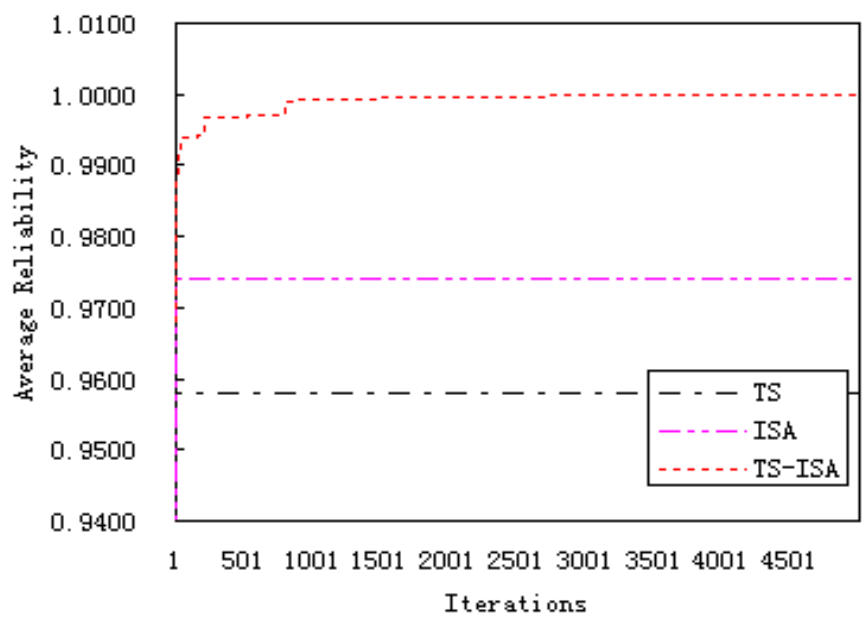

Figure 6. Overspeed Protection System

From the table 4, table 5 and table 6 , It can be clearly seen that the best results, the worst results, and the means results got by TS-ISA method are better than those got by TS and ISA. The best result value of TS-ISA is respectively $0.9999766491,0.9998896376$ and 0.9999546747 . The standard deviation (SD) is $1.8994 \mathrm{e}-06,1.3290 \mathrm{e}-07$ and $1.2506 \mathrm{e}-$ 06 respectively. These demonstrate that TS-ISA has a strong ability for getting the best results and stability than the TS and ISA.

Form Figure 4, Figure 5 and Figure 6, the trends show that TS-ISA has a better result and a faster convergence rate than the other two approaches.

Above all, the proposed TS-ISA method is effective and is superior to other methods for reliability optimization problems.

\section{Conclusions}

In this paper, a mixed TS-ISA algorithm is proposed to solve the RRAP. The presented algorithm benefits from merit of Tabu search approach and interior search method. Simulation experiments shows that proposed TS-ISA algorithm is effective, efficient and superior to other methods in literatures. Future work is using the TS-ISA algorithm to solve other more complex mixed integer programming problem. 


\section{Acknowledgment}

This work supported by the Maor International Joint Research Program of China (Grant No. 2014DFB70120).

\section{References}

[1] W. Kuo, and V.R. Prasad, "An annotated overview of system-reliability optimization,” IEEE Transaction on Reliability, vol. 49, no. 2, (2000).

[2] Yi-Chih Hsieh, Ta-Cheng Chen, and Dennis L. Bricker, "Genetic algorithms for reliability design problems," Microelectronics Reliability, vol. 38, no. 10, (1998).

[3] P.S. You, and T.C.Chen, "An efficient heuristic for series-parallel redundant reliability problems," Computers and Operations Research, vol. 32, no. 8, (2005).

[4] Y.C. Liang, and Y.C. Chen, "Redundancy allocation of series-parallel systems using a variable neighborhood search algorithm," Reliability Engineering and System Safety, vol. 92, no. 8, (2007).

[5] L dos Santos Coelho, "An efficient particle swarm approach for mixed-integer programming in reliability-redundancy optimization applications," Reliability Engineering and System Safety, vol. 94,no. 4, (2009).

[6] H Garg, and S.P. Sharma, "Multi-objective reliability-redundancy allocation problem using particle swarm optimization," Computers \& Industrial Engineering, vol. 64, no. 1, (2013).

[7] L Dallegrave Afonso, V Cocco Mariani, and L dos Santos Coelho, "Modified imperialist competitive algorithm based on attraction and repulsion concepts for reliability-redundancy optimization," Expert Systems with Applications, vol. 40, no. 9, (2013).

[8] N Safaei, R Tavakkoli-Moghaddamb, and C Kiassat, "Annealing-based particle swarm optimization to solve the redundant reliability problem with multiple component choices," Applied Soft Computing, vol. 12 , no. 11, (2012).

[9] L Wang, and L-p Li, "A coevolutionary differential evolution with harmony search for reliabilityredundancy optimization," Expert Systems with Applications, vol. 39, no. 5, (2012).

[10] S-M Chen, A Sarosh, Y-F Dong, "Simulated annealing based artificial bee colony algorithm for global numerical optimization," Applied Mathematics and Computation, vol. 219, no. 8, (2012).

[11] L Xiong, S-g Gong, "Reliability Allocation of Underwater Experiment System Based on Particle Swarm Optimization," Journal of Networks, vol. 8, no. 6, (2013).

[12] M. Hikita, Y. Nakagawa, and H. Harihisa, "Reliability optimization of systems by a surrogate constraints algorithm," IEEE Trans. Reliab, vol. 41, no. 3, (1992).

[13] K. Gopal, K. Aggarwal, and J.S. Gupta, "An improved algorithm for reliability optimization,” IEEE Trans. Reliab, vol. 27, no. 5, (1978).

[14] T. Yokota, M. Gen, and Y.X. Li, "Genetic algorithm for non-linear mixed integer programming problems and its applications," Comput. Ind. Eng, vol. 30, no. 4, (1996).

[15] A K. Dhingra, "Optimal Apportionment of Reliability \& Redundancy in Series Systems Under Multiple Objectives," IEEE Transactions on Reliability, vol. 41,no. 4, (1992).

[16] A H. Gandomi. "Interior search algorithm (ISA): A novel approach for global optimization", ISA Transactions, vol. 53, no. 4, (2014). 